\title{
Recent Advances in the Field of Vertical-External-Cavity Surface- Emitting Lasers
}

\author{
Arash Rahimi-Iman, ${ }^{\text {a }}$ Mahmoud Gaafar, ${ }^{\text {a }}$ Dalia Al Nakdali, ${ }^{\text {a }}$ Christoph Möller, ${ }^{\text {a }}$ Fan Zhang, \\ Matthias Wichmann, ${ }^{a}$ Mohammad Khaled Shakfa, ${ }^{a}$ Ksenia A. Fedorova, ${ }^{\mathrm{b}}$ Wolfgang Stolz, ${ }^{\mathrm{a}, \mathrm{c}}$ \\ Edik U. Rafailov, ${ }^{\mathrm{b}}$ and Martin $\mathrm{Koch}^{\mathrm{a}}$ \\ ${ }^{a}$ Department of Physics and Material Sciences Center, Philipps-Universität Marburg, Renthof 5, D- \\ 35032 Marburg, Germany; \\ ${ }^{\mathrm{b}}$ Optoelectronics and Biomedical Photonics Group, School of Engineering and Applied Science, \\ Aston University, Aston Triangle, Birmingham B4 7ET, UK; \\ 'NAsP III/V GmbH, Am Knechtacker 19, 35041 Marburg, Germany; \\ *arash.rahimi-iman@physik.uni-marburg.de
}

\begin{abstract}
Vertical-external-cavity surface-emitting lasers (VECSELs) have proved to be versatile lasers which allow for various emission schemes which on the one hand include remarkably high-power multi-mode or single-frequency continuouswave operation, and on the other hand two-color as well as mode-locked emission. Particularly, the combination of semiconductor gain medium and external cavity provides a unique access to high-brightness output, a high beam quality and wavelength flexibility. Moreover, the exploitation of intra-cavity frequency conversion further extends the achievable radiation wavelength, spanning a spectral range from the UV to the THz. In this work, recent advances in the field of VECSELs are summarized and the demonstration of self-mode-locking (SML) VECSELs with sub-ps pulses is highlighted. Thereby, we present studies which were not only performed for a quantum-well-based VECSEL, but also for a quantum-dot VECSEL.
\end{abstract}

Keywords: Semiconductor disk lasers, vertical-external-cavity surface-emitting laser, self-mode-locking, mode-locked lasers.

\section{INTRODUCTION}

Vertical-external-cavity surface-emitting lasers (VECSELs), also known as semiconductor disk lasers (SDLs), are versatile lasers which serve as an excellent platform for the realization of various emission schemes. Following the first demonstration in 1997 by Kuznetsov et al. [1], a wide range of modifications and improvements towards more specific applications were conducted. Optimizations based on microscopic modeling [2] and a detailed analysis of the thermal impedance [3, 4] have pushed the output power of SDLs beyond 100W [5]. Owing to their remarkable design flexibility and features, not only the realization of high-power multi-mode [5,6] or single-frequency [7] continuous-wave operation schemes have been pushed forward in recent years, but also the accomplishment of dual-color [8] as well as modelocked $[9,10]$ operation. Moreover, in order to expand the accessible wavelength range drastically, their external resonator is well exploited for intra-cavity frequency conversion processes via nonlinear elements: while secondharmonic generation pushes the boundaries of the emission wavelength into the UV [11], terahertz frequencies can be reached by difference-frequency generation inside a two-color VECSEL $[12,13]$. This is to name a few important achievements in this field.

VECSELs have particularly attracted much attention within the last decade as alternative sources of pulsed laser light, since they can typically provide both a high output power and an outstanding beam quality. The demonstration of the first mode-locked (ML) VECSEL, which was achieved by Hoogland et al. for a central wavelength of $1 \mu \mathrm{m}$ with $22 \mathrm{ps}$ long pulses [14], dates back to the year 2000 and kicked off a movement towards an entering of the fs-pulse-regime. Additionally, the exploration of pulsed operation for a variety of wavelength has been on the agenda. It did not take long

Vertical External Cavity Surface Emitting Lasers (VECSELs) V, edited by Mircea Guina, Proc. of SPIE Vol. 9349, 934906 - (c) 2015 SPIE - CCC code: 0277-786X/15/\$18 - doi: 10.1117/12.2079182 
that VECSELs - devices which are also compact and robust - were considered becoming cost-efficient alternatives to commercial mode-locked lasers with short pulses, high peak powers and enhanced tenability.

Up-to-date, mode-locking of VECSELs required using resonator-integrated [9, 15] or chip-integrated [16] semiconductor saturable-absorber mirrors (SESAMs). Indeed, besides semiconductor materials, saturable absorbers as graphene [17, 18] and carbon nanotubes [19] have also been employed for ML operation of VECSELs. However, the power-sensitive, complex and costly absorber mirrors, which have to be carefully designed for a certain wavelength range, naturally impose limitations on the device's performance. Fortunately, on the other hand, mode-locking has also been reported to take place without any additional saturable absorber in the system - an effect called self-mode locking (SML) [20-25].

In this context, we highlight recent demonstrations of SESAM-free VECSELs which are operated under SML conditions in this work. Thereby, SML operation is not only presented for a quantum-well VECSEL [22, 25], but also for a quantum-dot device [23]. Furthermore, passively harmonically self-mode-locked devices with sub-ps-pulsed operation are shown which run at discrete power levels up to the third harmonic [22]. However, it is worth to note that the mechanism behind this effect has not been fully understood, yet, and future investigations will provide a broader picture with respect to this feature.

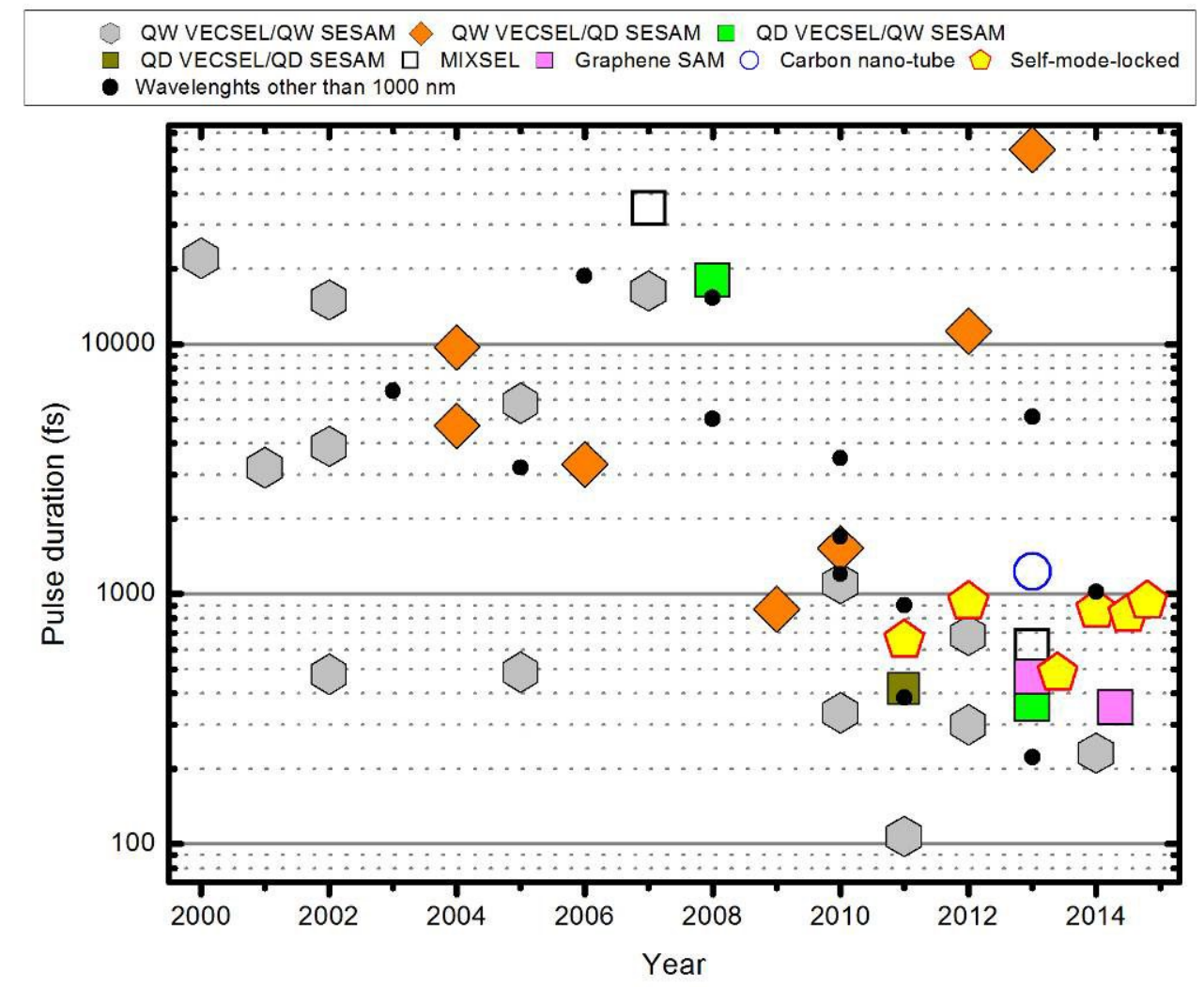

Figure 1. Pulse durations of mode-locked optically pumped VECSELs. Big symbols represent devices emitting around a wavelength of $1 \mu \mathrm{m}$, while small black dots represent devices with wavelengths other than $1 \mu \mathrm{m}$.

\section{GAIN MIRROR DESIGNS}

In the following, a brief overview on the design of the employed chip structures is given. Structure 1 is a MOVPE grown VECSEL chip with 10 (InGa)As quantum wells (QWs) equally spaced by $\lambda / 2$ (GaP)As barrier layers. The distributed Bragg reflector (DBR) consists of $24 \frac{1}{2}$ pairs of $\lambda / 4 \mathrm{GaAs} /(\mathrm{AlGa}) \mathrm{As}$ layers and is transparent to the wavelength of the fibre-coupled $808 \mathrm{~nm}$ diode pump laser. The structure was grown "bottom- 
up" and flip-chip bonded with Au-In solid-liquid interdiffusion onto a $350 \mu \mathrm{m}$-thick CVD diamond heat spreader. Originally, the chip was designed as a resonant chip and exhibits the micro-cavity resonance at the quantum well gain peak at $1010 \mathrm{~nm}$. For the purpose of mode-locking, the cap layer thickness was reduced from $\lambda / 2$ to $\lambda / 4$ via wet etching in order to obtain an anti-resonant micro-cavity with minimized group delay dispersion (GDD) and a spectrally broadened effective gain of the structure. A schematic drawing of the VECSEL chip's structure is shown in Fig. 2(a).

Structure 2 was grown by molecular-beam epitaxy. It consists of 35 layers of Stranski-Krastanow grown InGaAs QDs within GaAs spacers, organized as five stacks of seven QD layers. These stacks are distributed within the cavity to be located at standing-wave electric field antinodes of the optical mode for an optimum gain. As in structure 1, a ternary DBR was grown, which consists of 29.5 pairs of GaAs/(AlGa)As and is transparent to the wavelength of the fiber-coupled $808 \mathrm{~nm}$ pump laser. Both, the QDs and the DBR, are designed for an emission wavelength of $1040 \mathrm{~nm}$. A schematic drawing of the VECSEL chip's structure is shown in Fig. 2(b). The VECSEL chip is capillary bonded to an intra-cavity diamond heat spreader which exhibits an anti-reflection coating. The anti-reflection coating is also minimizing the GDD at the design wavelength.

Table 1. Overview of the semiconductor disk laser gain mirrors used in this work.

\begin{tabular}{lcc}
\hline & Structure 1 & Structure 2 \\
\hline Wavelength (nm) & 1010 & 1040 \\
Gain type & QW & QD \\
Gain layers & 10 single QWs & 35 QD layers, in stacks of 7 \\
Mirror pairs per DBR & 24.5 & 29.5 \\
Resonance & Anti-resonant & Anti-resonant \\
Heat management & Flip-chip bonded onto diamond & "Top emitter" with intra-cavity \\
& heat spreader & diamond heat spreader \\
\hline
\end{tabular}

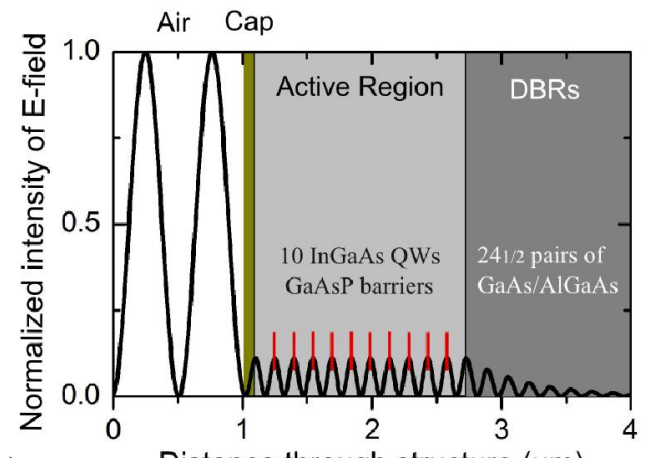

(a)

Distance through structure $(\mu \mathrm{m})$

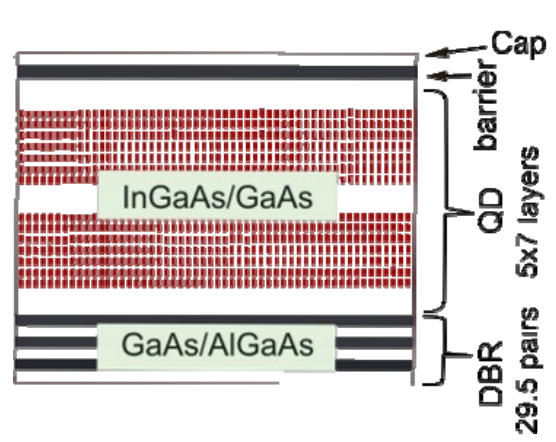

(b)

Figure 2. (a) Electric-field distribution of a standing optical wave inside the near-antiresonant gain structures normalized to the input intensity in the case of the QW-gain mirror (structure 1). The position of the quantum wells are represented by the vertical red lines. (b) Scheme of the QD-gain mirror structure. 5 Stacks consisting of 7 layers Stransky-Krastanow-grown QDs are incorporated into the active region of the chip (structure 2).

\section{EXPERIMENTAL SETUPS}

Pulsed VECSELs, similar to cw ones, employ the gain mirror in combination with an external cavity, which is defined by at least one mirror. While the cavity geometry of the presented SML VECSELs varies slightly, the principle which 
allows for mode-locking remains the same, as mentioned later. Here, the laser setups for a quantum well (QW) and quantum dot (QD) VECSEL are schematically shown in Fig. 3.

As the diamond heat spreader of the QW-VECSEL is attached to the chip's DBR structure, the VECSEL chip is directly mounted on a water-cooled copper heat sink. The laser resonator can be seen as a Z-shaped cavity, which is formed by a flat output coupler (OC) with a transmittance of $1.6 \%$, the gain chip itself, and a highly reflective (HR) curved mirror $(\mathrm{CM})$ with a radius of curvature (RC) of $150 \mathrm{~mm}$ as well as a plane HR mirror. With a total cavity length amounting to $30 \mathrm{~cm}$, a free spectral range of approximately $0.5 \mathrm{GHz}$ is determined. The angle of incidence on the curved mirror was kept below $10^{\circ}$ in order to avoid excessive astigmatism. The cavity is optimized for mode-locked operation assuming Kerr-lensing inside the VECSEL chip structure. Therefore, a variable slit is placed directly in front of the HR end mirror (cf Fig. 3(a)). An $808 \mathrm{~nm}$ fiber-coupled diode laser which can deliver up to $35 \mathrm{~W}$ output power is used to pump the system.

For the QD-VECSEL setup, the copper heat sink is attached to both the outer region of the intracavity diamond heat spreader on the top of the gain mirror and the DBR structure on the reverse side of the VECSEL chip. A thermoelectric cooler is employed to extract excess heat from the copper heat sink to the closed-cycle cooling water. To complete the laser cavity, a concave $(\mathrm{RC}=100 \mathrm{~mm}) \mathrm{OC}$ mirror with a transmittance of $0.6 \%$ is placed at a position $97 \mathrm{~mm}$ away from the gain chip. Thereby, a linear cavity is formed with a free spectral range of approximately $1 \mathrm{GHz}$. Similar to the QWVECSEL setup, a variable slit is inserted close to the external mirror in this linear cavity (cf. Fig. 3(b)). The pump laser system is the same as used in the QW-VECSEL setup.

(a)

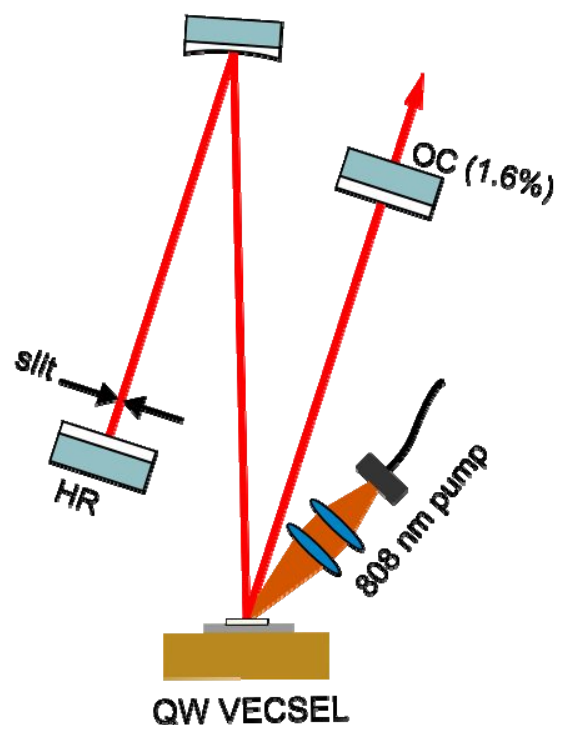

(b)

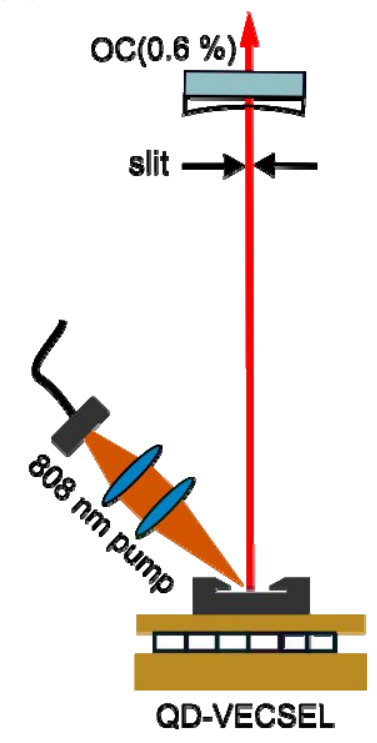

Figure 3. Schematic drawing of the setups used to realize SML (a) for a QW-VECSEL and (b) a QD-VECSEL. These two designs represent the common cavity configurations known as z-cavity and linear cavity, respectively.

\section{RESULTS}

\subsection{Quantum well VECSEL chip}

Self-mode-locking, which can be also named saturable-absorber-mirror-free mode-locking, is initiated in the VECSEL system when the slit in front of the HR end mirror is moved or the slit width is narrowed. Moreover, to stabilize SML operation, the cavity length was fine-tuned (see also [21, 22] for more details on the systems). The long-time-span pulse train of the SML QW VECSEL reveals stable operation on the microsecond scale (see Fig. 4(a)), with a repetition rate 
faster, while also on the order of Ti:Sapphire oscillators: A 500-MHz repetition rate is demonstrated for the fundamental mode in a close-up of the pulse train, shown in Fig. 4(b). Another indicator of stable pulsed operation can be obtained from RF spectra, of which an example is shown in Fig. 4(c) for the signal of the fundamental repetition rate. Here, the $\mathrm{RF}$ linewidth is less than $30 \mathrm{kHz}$, indicating stable SML operation. The mode-locked pulse duration was measured with a self-made intensity autocorrelator with a scan range of $\sim 130 \mathrm{ps}$. Figure $4(\mathrm{~d})$ presents a long-delay autocorrelation trace for fundamental mode-locking measured with a self-made intensity autocorrelator with a scan range of $\sim 130 \mathrm{ps,}$ confirming single-pulse operation. This measurement also demonstrates the absence of a pedestal. Typical short-delay autocorrelation traces, from which pulse durations are estimated, are shown in the following section (cf. Fig. 8(a'-c')).

(a)

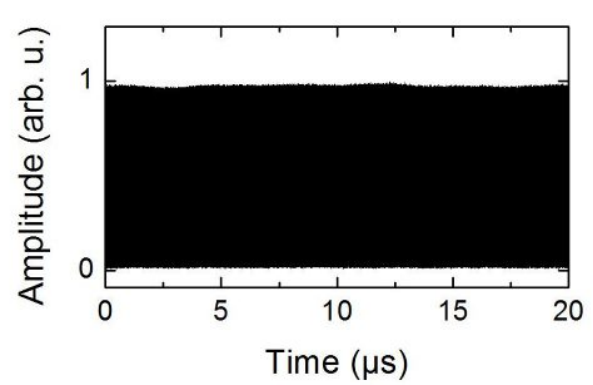

(c)

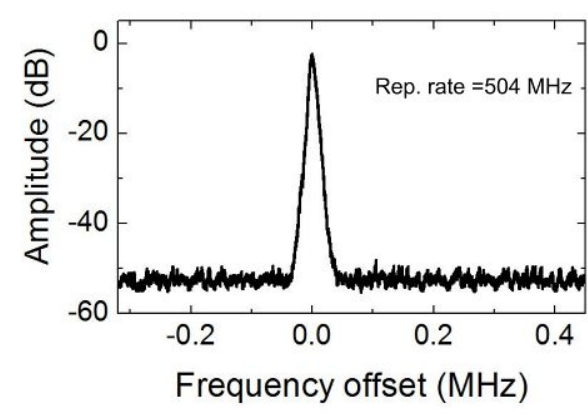

(b)

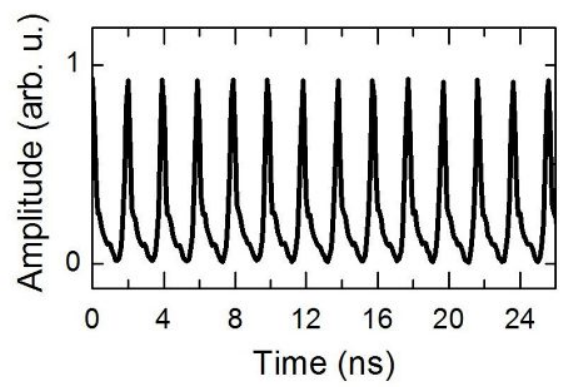

(d)

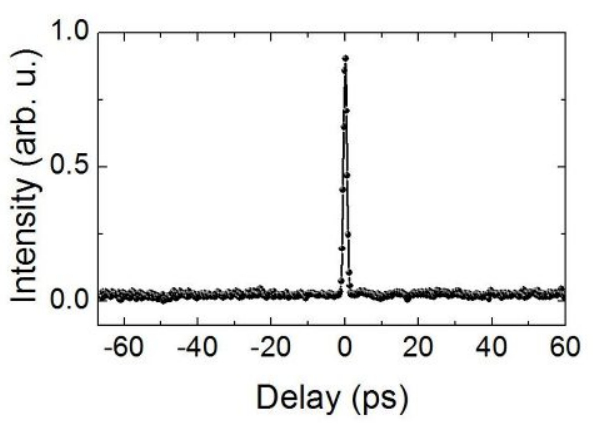

Figure 4. (a) Diagram of the long-time-span pulse train of the SML QW device. (b) A 500-MHz repetition rate is revealed for the fundamental mode in a close-up of the pulse train. (c) RF spectrum of the fundamental repetition rate. The RF linewidth is less than $30 \mathrm{kHz}$, indicating stable SML operation. (d) Single pulse operation is confirmed by a long-delay autocorrelation trace for fundamental mode-locking.

To provide further evidence of a mode-locked operation, a nonlinear frequency conversion in a BBO crystal is performed using the SML QW VECSEL. By directing the out-coupled laser beam of the VECSEL into the nonlinear crystal, green light is externally produced via second-harmonic generation (SHG) with the infrared pulsed laser light. While a clear spectrum of the SHG signal can be measured if the laser is mode-locked, in the case of continuous-wave operation no SHG signal is observed, owing to the fact that SHG is an intensity-dependent nonlinear effect. Depicted in Fig. 5, the experimental setup of the self-mode-locked QW VECSEL shows how SHG is utilized to produce green light externally. In the top inset of Fig. 5, a spectrum of the frequency-doubled SHG signal together with the spectrum of the original laser output is presented. Finally, a beam quality measurement confirms operation in the fundamental-transverse mode with $\mathrm{M}^{2}$ values less than 1.1 for both axes, as shown by the bottom insets of Fig. 5 (left: beam profile, right: $\mathrm{M}^{2}$ measurement). It is worth mentioning, that whenever a new mode-locking technique is demonstrated, the confirmation of mode-locked operation requires different measurements to be carried out, particularly beyond standard characterization methods which typically can't serve as an unambiguous evidence of mode-locking. Indeed, this has motivated part of our experiments and further discussion of the performed investigations and obtained results can be found in Ref. [25]. 


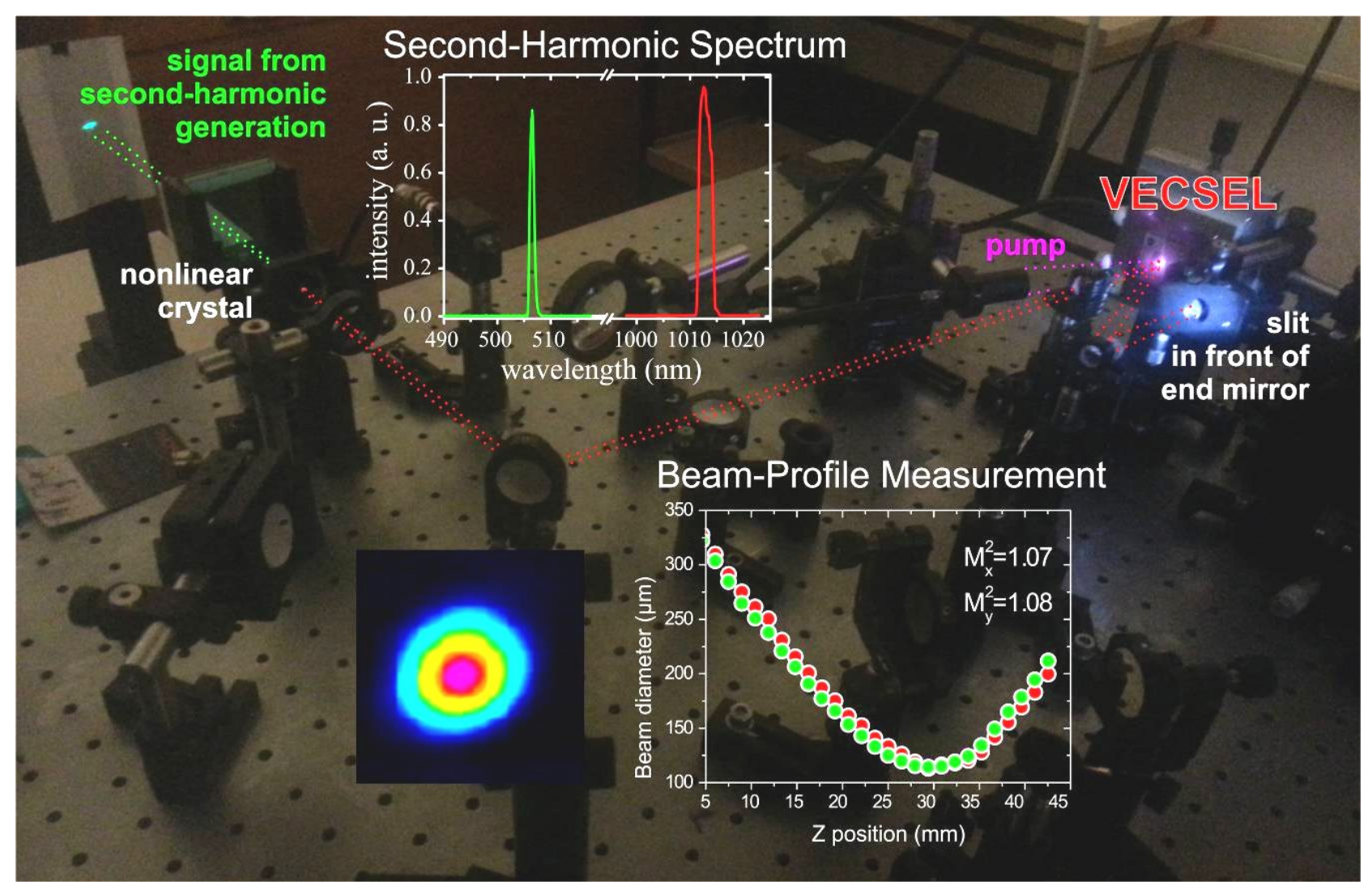

Figure 5. The experimental setup of the self-mode-locked QW VECSEL shows how second-harmonic generation is utilized to produce green light externally via the infrared pulsed laser light. Top inset: Spectrum of the frequency-doubled SHG signal and the original laser light. Bottom inset: Beam quality measurement confirming operation in fundamental-transverse mode with $\mathrm{M}^{2}$ values less than 1.1 for both axes.

\subsection{Harmonic mode-locking}

Next, we want to summarize the observation of harmonic self-mode-locking which can be achieved with the presented QW device. Interestingly, mode-locking is only observed for nearly discrete pump levels. The error bars in Fig. 6 represent the pump regions in which a stable ML operation was accomplished in repeated investigations. For the lowest pump level, fundamental mode-locking is observed. For the higher pump levels the repetition rate is doubled and tripled, corresponding to two and three equally spaced pulses in a single cavity roundtrip, respectively. In our observations, ML is not self-starting but can be initiated when the slit width is narrowed or the slit is moved. However, the fundamental mode-locked operation is self-sustaining and the slit can be opened completely without disturbing the ML process. In harmonic operation, a self-sustaining ML is only achieved with the slit partially closed.

In order to confirm stable mode-locking, an RF spectrum (cf. Fig. 7), an optical spectrum and an autocorrelation trace (cf. Fig. 8) was performed. The resulting time bandwidth products of 0.69 (first), 0.73 (second) and 0.72 (third) reveal that the pulses are not transform limited which can be attributed to a remaining GDD caused by the VECSEL chip. Also, we obtain the peak powers $948 \mathrm{~W}$ for the first, $752 \mathrm{~W}$ for the second and $754 \mathrm{~W}$ for the third harmonic ML. The nearly constant peak power for the different power levels indicates that a certain intra-cavity power is needed for the underlying ML mechanism. 


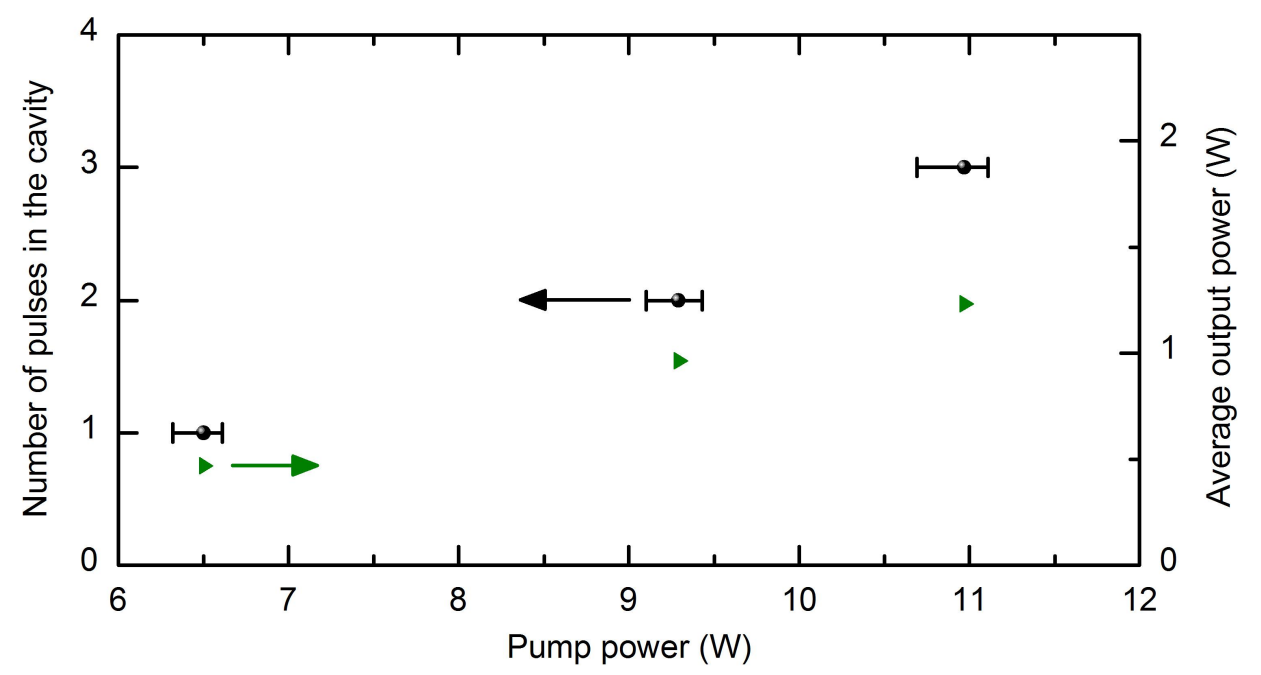

Figure 6. Number of pulses in the cavity as a function of the optical pump power (black squares, left axis). The average output power of the device is plotted with respect to the right axis (green circles).
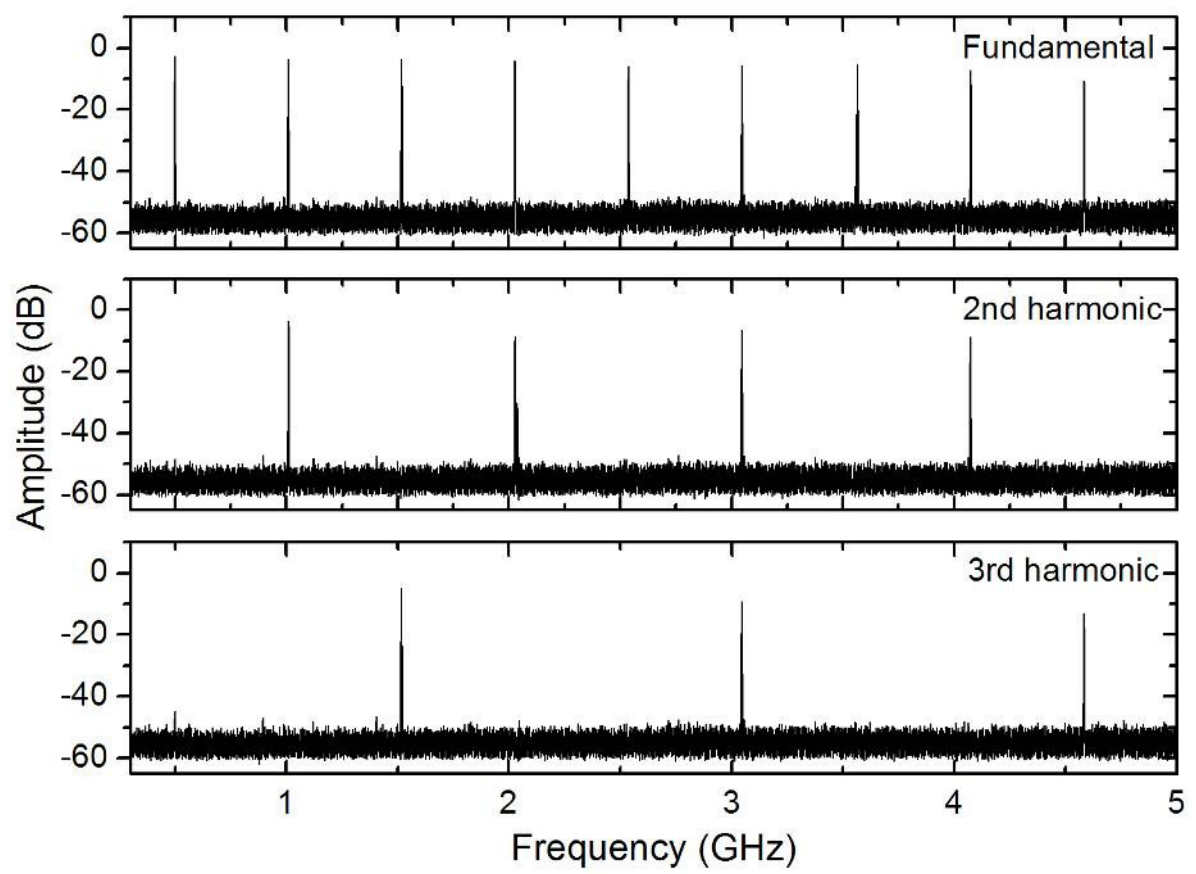

Figure 7. RF spectra reveal fundamental (top row), second harmonic (center) and third harmonic (bottom) mode-locking. 

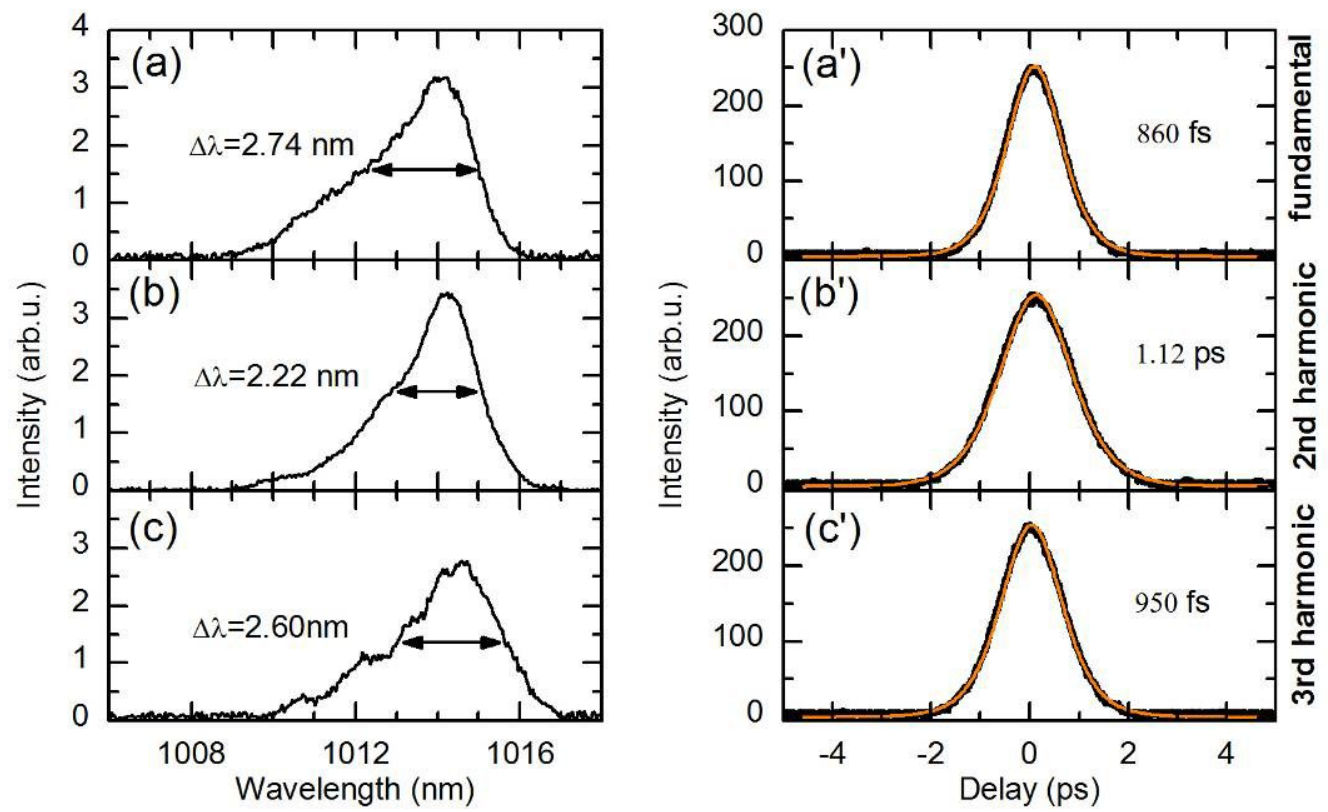

Figure 8. (a)-(c) Optical spectra for the SML VECSEL in the regimes of fundamental, second-harmonic and third-harmonic mode-locking, respectively. (a')-(c') Autocorrelation traces (black) with $\operatorname{sech}^{2}$ fits (orange) corresponding to signal shown in (a)-(c), respectively.

\subsection{Quantum dot VECSEL chip}

In this section, we present the first passively-self-mode-locked optically-pumped QD-VECSEL. The measurements indicated sub-picosecond duration of the laser pulses in the mode-locked regime with $750 \mathrm{~mW}$ average output power at $1.5 \mathrm{GHz}$ repetition rate. The RF spectrum which was measured over a span of $6.5 \mathrm{GHz}$ using a $100 \mathrm{kHz}$ resolution bandwidth (RBW), is shown in Fig. 9(a). Moreover, Fig. 9(b) shows an RF spectrum of the fundamental repetition rate measured over a span of $1 \mathrm{MHz}$ using a RBW of $10 \mathrm{kHz}$. Here, a clear peak at $1.5 \mathrm{GHz}$ with a signal to noise ratio of about $45 \mathrm{~dB}$ is observed.

Fig. 10(a) presents the corresponding optical spectrum of the SML QD-VECSEL at room temperature centered at 1038 $\mathrm{nm}$. The mode-locked pulse duration is yielded assuming $\mathrm{sech}^{2}$ shaped pulses in the autocorrelation trace representatively shown in Fig. 10 (b). In a temperature dependence study, pulse durations varied between approximately 0.8 and $1.0 \mathrm{ps}$.
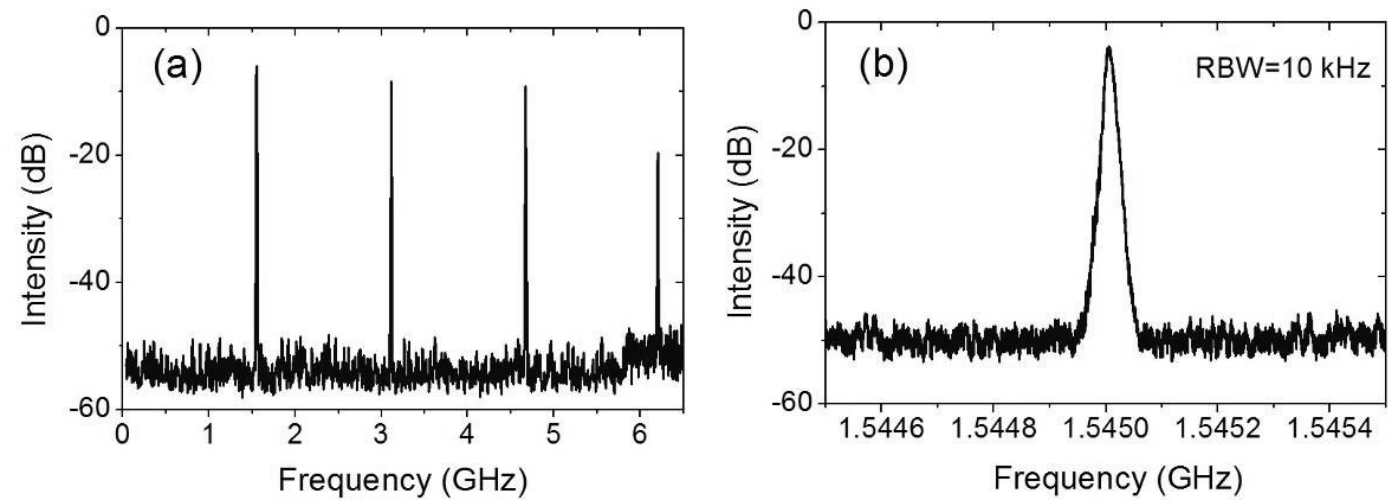

Figure 9. RF spectra measured over (a) a span of $6.5 \mathrm{GHz}$ and a RBW of $100 \mathrm{kHz}$, showing the first 4 harmonics, and (b) a span of $1 \mathrm{MHz}$ and a RBW of $10 \mathrm{kHz}$, with the RF signal centered around $1.545 \mathrm{GHz}$. 

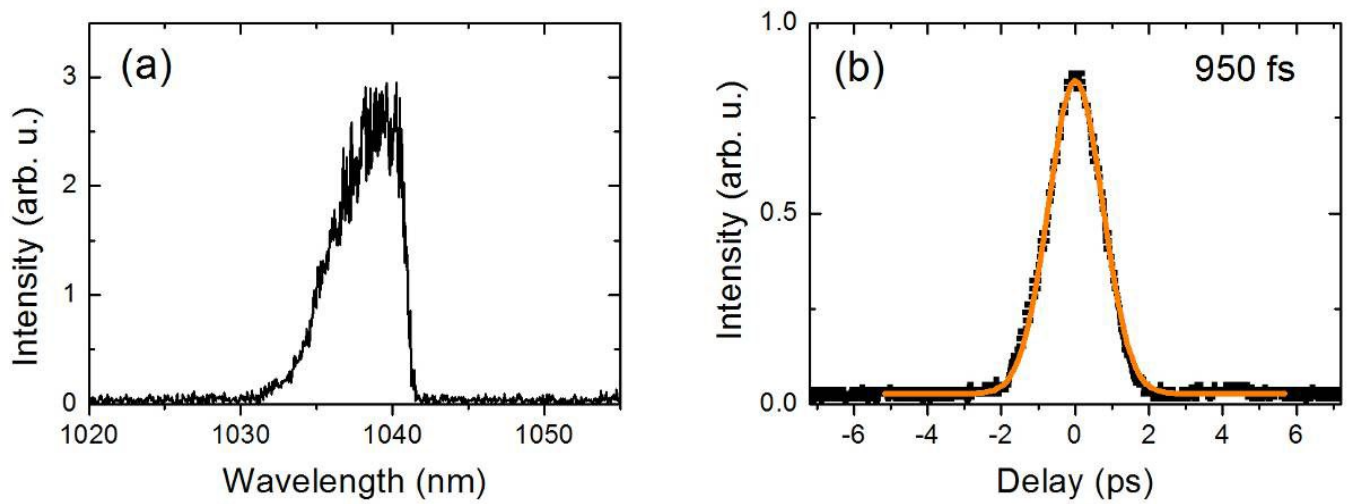

Figure 10. (a) Optical spectrum of the SML QD-VECSEL. (b) Corresponding autocorrelation trace. Black dotted: experimental data. Orange line: fit curve assuming a $\operatorname{sech}^{2}$ pulse.

\section{CONCLUSIONS}

In summary, we presented recent developments in the field of semiconductor disk lasers, well known as VECSELs, which provide access to various operation modes and features for a broad wavelength range. In addition to high-power operation, frequency stabilization and frequency conversion techniques, the field of mode-locking of VECSELs gained much attraction in recent years, with the efforts of the community leading towards an alternative source for pulsed lasers. In this context, we highlight the demonstration of self-mode-locking VECSELs as a variation of mode-locked VECSELs which do not require a saturable absorber mirror for pulsed operation, and thus circumvent some limitations set by saturable absorbers. Results are presented for a quantum well and a quantum dot device to emphasize that this quite young technique, even though the mechanism behind the effect has yet not been well understood, is capable of enabling mode-locked operation for different gain media as well as cavity configurations.

\section{ACKNOWLEDGEMENTS}

The authors acknowledge financial support by the DFG (GRK 1782 and SFB 1083) and EU FP7 program through FAST-DOT project (contract No. 224338). M. Gaafar acknowledges support from the Yousef Jameel scholarship funds. The authors would like to thank Innolume GmbH for the fabrication of the QD structures and Prof. O.G. Okhotnikov from Tampere University of Technology for the preparation of the QD-VECSELs.

\section{REFERENCES}

[1] Kuznetsov, M., Hakimi, F., Sprague, R. and Mooradian, A., "High-power ( $>0.5-\mathrm{W}$ CW) diode-pumped verticalexternal-cavity surface-emitting semiconductor lasers with circular TEM00 beams," IEEE Photonics Technol. Lett. 9, 1063-1065 (1997).

[2] Wang, T.-L., Heinen, B., Hader, J., Dineen, C., Sparenberg, M., Weber, A., Kunert, B., Koch, S. W., Moloney, J. V., Koch, M., and Stolz, W., "Quantum design strategy pushes high-power vertical-external-cavity surface-emitting lasers beyond 100 W," Laser Photonic Rev., 6(5), L12-L14 (2012)

[3] Hader, J., Wang, T.-L., Moloney, J. V., Heinen, B., Koch, M., Koch, S. W., Kunert, B., and Stolz, W., "On the measurement of the thermal impedance in vertical-external-cavity surface-emitting lasers," J. Appl. Phys., 113(15), 153102 (2013).

[4] Heinen, B., Zhang, F., Sparenberg, M., Kunert, B., Koch, M., and Stolz, W., "On the Measurement of the Thermal Resistance of Vertical-External-Cavity Surface-Emitting Lasers (VECSELs)," IEEE J. Quantum Electron., 48(7), 934-940 (2012). 
[5] Heinen, B., Wang, T. L., Sparenberg, M., Weber, A., Kunert, B., Hader, J., Koch, S. W., Moloney, J. V., Koch, M. and Stolz, W., "106 W continuous-wave output power from vertical-external-cavity surface-emitting laser," Electron. Lett. 48 (9), 516-517 (2012).

[6] Al Nakdali, D., Shakfa, M. K., Gaafar, M., Butkus, M., Fedorova, K. A., Zulonas, M., Wichmann, M., Zhang, F., Heinen, B., Rahimi-Iman, A., Stolz, W., Rafailov, E. U., and Koch M., "High-Power Quantum-Dot VerticalExternal-Cavity Surface-Emitting Laser Exceeding 8 W," IEEE Photonics Technol. Lett. 26(15), 1561 (2014)

[7] Zhang, F., Heinen, B., Wichmann, W., Möller, C., Kunert, B., Rahimi-Iman, A., Stolz, W. and Koch, M., "A 23watt single-frequency vertical-external-cavity surface-emitting laser," Opt. Express 22, 12817-12822 (2014).

[8] Wichmann, M., Shakfa, M. K., Zhang, F., Heinen, B., Scheller, M., Rahimi-Iman, A., Stolz, W., Moloney, J. V., Koch, S. W. and Koch, M., "Evolution of multi-mode operation in vertical-external-cavity surface-emitting lasers," Opt. Express 21(26) 31940 (2013).

[9] Keller, U. and Tropper, A. C., "Passively modelocked surface-emitting semiconductor lasers," Physics Reports 429, 67-120 (2006).

[10] Moloney, J.V., Kilen, I., Bäumner, A., Scheller, M., and Koch, S.W., "Nonequilibrium and thermal effects in modelocked VECSELS," Opt. Express 22(6), 6422-6427 (2014).

[11]Chen, Y. F., Lee, Y. C., Liang, H. C., Lin, K. Y., Su, K. W. and Huang, K. F., "Femtosecond high-power spontaneous mode-locked operation in vertical-external cavity surface-emitting laser with gigahertz oscillation," Opt. Lett. 36(23), 4581-4583 (2011).

[12] Scheller, M., Yarborough, J. M., Moloney, J. V., Fallahi, M., Koch, M., and Koch, S. W., "Room temperature continuous wave milliwatt terahertz source," Opt. Express 18(26), 27112-27117 (2010).

[13] Wichmann, M., Stein, M., Rahimi-Iman, A., Koch, S. W., and Koch, M., "Interferometric Characterization of a Semiconductor Disk Laser driven Terahertz Source," J. Infrared Milli. Terahz. Waves 35(6-7), 503-508 (2014).

[14] Hoogland, S., Dhanjal, S., Tropper, A. C., Roberts, S. J., Häring, R., Paschotta, R., and Keller, U., "Passively modelocked diode-pumped surface-emitting semiconductor laser," IEEE Photon. Technol. Lett. 12(9), 1135-1137 (2000).

[15] Wilcox, K.G., Tropper, A.C., Beere, H.E., Ritchie, D.A., Kunert, B., Heinen, B., Stolz, W., "4.35 kW peak power femtosecond pulse mode-locked VECSEL for supercontinuum generation," Opt. Express 21(2), 1599-1605 (2013)

[16] Mangold, M., Wittwer, V. J., Zaugg, C. A., Link, S. M., Golling, M., Tilma, B. W., and Keller, U., "Femtosecond pulses from a modelocked integrated external-cavity surface emitting laser (MIXSEL)," Opt. Express 21(21), 24904-24911 (2013).

[17]Zaugg, C. A., Sun, Z., Wittwer, V. J., Popa, D., Milana, S., Kulmala, T. S., Sundaram, R. S., Mangold, M., Sieber, O. D., Golling, M., Lee, Y., Ahn, J. H., Ferrari, A. C., and Keller, U., "Ultrafast and widely tuneable verticalexternal-cavity surface-emitting laser, mode-locked by a graphene-integrated distributed Bragg reflector," Opt. Express 21(25), 31548-31559 (2013).

[18] Husaini, S., and Bedford, R. G., "Graphene saturable absorber for high power semiconductor disk laser modelocking," Appl. Phys. Lett. 104(16), 161107 (2014).

[19] Seger, K., Meiser, N., Choi, S. Y., Jung, B. H., Yeom, D.-I., Rotermund, F., Okhotnikov, O., Laurell, F., and Pasiskevicius, V., "Carbon nanotube mode-locked optically-pumped semiconductor disk laser," Opt. Express 21(15), 17806-17813 (2013).

[20] Kornaszewski, L., Maker, G., Malcolm, G. P. A., Butkus, M., Rafailov, E. U. and Hamilton, C. J., "SESAM-free mode-locked semiconductor disk laser," Laser Photonics Rev. 6(6), L20-L23 (2012).

[21] Albrecht, A. R., Wang, Y., Ghasemkhani, M., Seletskiy, D. V., Cederberg, J. G. and Sheik-Bahae, M., "Exploring ultrafast negative Kerr effect for mode-locking vertical external-cavity surface-emitting lasers," Opt. Express 21(23), 28801-28808 (2013).

[22] Gaafar, M., Möller, C., Wichmann, M., Heinen, B., Kunert, B., Rahimi-Iman, A., Stolz, W. and Koch, M., "Harmonic self-mode-locking of optically pumped semiconductor disc laser," Electron. Lett. 50(7), 542-543 (2014).

[23] Gaafar, M., Al Nakdali, D., Möller, C., Fedorova, K. A., Wichmann, M., Shakfa, M. K., Zhang, F., Rahimi-Iman, A., Rafailov, E. U. and Koch, M., "Self-mode-locked quantum-dot vertical-external-cavity surface-emitting laser," Opt. Lett. 39(15), 4623-4626 (2014).

[24] Liang, H. C., Tsou, C. H., Lee, Y. C., Huang, K. F. and Chen, Y. F., "Observation of self-mode-locking assisted by high-order transverse modes in optically pumped semiconductor lasers," Laser Phys. Lett. 11, 105803 (2014).

[25] Gaafar, M., Richter, P., Keskin, H., Möller, C., Wichmann, M., Stolz, W., Rahimi-Iman, A. and Koch, M., "Selfmode-locking semiconductor disk laser," Opt. Express 22(23), 28390-28399 (2014). 